\title{
LA PROBLEMÁTICA DE LA COMUNICACIÓN INTERCULTURAL: CONSIDERACIONES DIDÁCTICAS PARA LA ENSEÑANZA DE LAS LENGUAS
}

\begin{abstract}
Małgorzata Spychała, La problemática de la comunicación intercultural: consideraciones didácticas para la enseñanza de las lenguas [The problems of intercultural communication: didactic considerations for teaching languages], Studia Romanica Posnaniensia, Adam Mickiewicz University Press, Poznań, vol. XLI/1: 2014, pp. 87-100. ISBN 978-83-232-2673-4. ISSN 0137-2475. eISSN 20844158. Doi: $10.7169 /$ strop2014.411.006
\end{abstract}

The coexistence of different nations, races i.e. cultures in the same social space that could be a country, a village or even a company is already a fact. Nowadays, not taking into account the tools of intercultural communication may result in damaging every communicative act. We believe that there is a necessity for revising and explaining the concepts related to the subject of intercultural communication from the perspective of foreign language teaching. In this article we will discuss such concepts as: interculturalism, transculturation, acculturation, integration, identity, etc. We will also look into the origin of intercultural communication. All these reflections will bring us closer to the intercultural competence which, in our opinion, students of foreign languages, i.e. intercultural speakers, should possess. In conclusion, we present the main objectives of the Common Frame of Reference for the Languages of the Council of Europe (2002) with regard to intercultural education.

Keywords: intercultural communication, intercultural competence, interculturalism, transculturation, acculturation

\section{EL ORIGEN Y LA SITUACIÓN ACTUAL DE LA COMUNICACIÓN INTERCULTURAL. CLARIFICACIÓN CONCEPTUAL Y TERMINOLÓGICA}

Aunque la comunicación intercultural es un campo de estudio nuevo, se puede decir que, por ejemplo en el ámbito hispano, los primeros encuentros interculturales empezaron con el descubrimiento de América Latina ${ }^{1}$. Con la llegada de los españoles, los pueblos indígenas tuvieron que asimilarse a la cultura de la potencia colonizadora. La sociedad colonial estaba fracturada en un sistema de castas semejante al feudalismo europeo. También se estableció una nueva estructura social que era difícil de entender para los indígenas. Se intentó destruir todos los vestigios de la religión indígena, la lengua y su cultura. Actualmente, los investigadores e historiadores consideran

\footnotetext{
${ }^{1}$ Véase Szurmuk y Mckee Irvin (2009: 119).
} 
que éste fue uno de los grandes crímenes de la historia cultural, que posteriormente ha dificultado los esfuerzos realizados por entender la asombrosa cultura de aquellos pueblos.

Un profundo cambio se produjo después de la Segunda Guerra Mundial con los procesos de descolonización. Los interlocutores se dieron cuenta de que para poder comunicarse y al mismo tiempo conseguir éxito en las negociaciones era imprescindible conocer la cultura de los distintos pueblos. Más tarde, la creación de diferentes organizaciones internacionales (ONU, Organización Mundial de la Salud, UNESCO, etc.) mostró que verdaderamente existe una necesidad de comunicarse mejor entre diferentes sociedades culturales. Basta recordar que en los años 50 fue EE.UU, ya una potencia económica, la que llamó la atención sobre la comunicación intercultural. De hecho uno de los primeros investigadores que por primera vez utilizó la expresión "intercultural communication" fue el antropólogo norteamericano Edward T. Hall, cuyo libro titulado «The Silence Language» fue publicado en $1959^{2}$.

Posteriormente, un gran número de investigadores (Gudykunts, Kim 1984; Byram y Flaming 1998; Kramsch, 1998; Murdoch, 1999; Ting-Toomey, S.1999; Matsumoto, Juang, 2007 y otros) empezaron a tratar el asunto de la comunicación intercultural desde distintas perspectivas. Vieron que en esa década de creciente migración ya no era suficiente el dominio de la lengua del país meta para evitar los posibles conflictos producidos por la incomprensión. En la actualidad, desde la Unión Europea, María Cristina Bravo Bosch (1998: 143) ha apuntado que esta se transformará en una «aldea global» que al principio será una unión puramente técnica, organizativa, económica «hasta que todos nos sintamos, aún con nuestras diferencias, ciudadanos de un mismo Estado».

Al principio de nuestras reflexiones debemos hacer una clarificación terminológica de los conceptos que aparecen cuando se habla de la comunicación intercultural. Empezaremos por los dos adjetivos más renombrados, multicultural e intercultural. Para explicar las dos nociones decidimos recurrir a la distinción hecha por el Consejo de Europa. Su representante, Lotty van den Berg-Eldering (1987: 20), explica que el concepto multicultural se refiere a la presencia, en una sociedad, de dos o más culturas. En cambio, intercultural se emplea para designar la acción y la comunicación: la comunicación entre individuos, entre grupos, y entre instituciones pertenecientes a culturas diferentes o procedentes de culturas diferentes que normalmente no se relacionan entre sí.

Isabel Iglesias Casal (1998: 469) ${ }^{3}$ afirma que los encuentros interculturales se relacionan con posturas o actitudes no necesariamente positivas, que muchas veces nos dejan «caer en el estereotipo porque éste nos proporciona una imagen mental simplificada sobre los miembros de un grupo compartida socialmente». Se trata sobre

2 Véase Rodrigo Alsina (1999: 23).

${ }^{3}$ Seguimos a Iglesias Casal (1998) que se basa en las definiciones que recoge Colectivo Amani, «Educación intercultural. Análisis y resolución de conflictos», Madrid, Edit. Popular(1994: 134-137). 
todo del etnocentrismo y relativismo cultural. Con la postura etnocéntrica analizamos a los miembros de otra cultura desde la perspectiva cultural del país de origen y no somos capaces de realizar un acto comunicativo con rasgos de tolerancia y empatía. En cambio, el relativismo cultural «propone el conocimiento y análisis de otras culturas desde sus propios valores, estableciendo, además la igualdad de todas ellas. A esta actitud, en apariencia respetuosa y tolerante, le falta la búsqueda del encuentro entre culturas: Yo te respeto, te comprendo, pero tú en tu casa y yo en la mía (Iglesias Casal, 1998: 469).

Es importante hablar también de otros conceptos que además de ser relevantes para los países hispanoamericanos siguen estando vigentes en el contexto intercultural. Se trata de: mestizaje, criollización y transculturación. El mestizaje, como sabemos, es un concepto latinoamericano que originalmente se refería al resultado de la unión de los españoles con los indios o negros. Hoy en día este concepto acoge nuevos significados, en su mayoría positivos. Por ejemplo, los autores hispanoamericanos cuando usan esta noción piensan en los intercambios que se producen en el ámbito mundial. Sin embargo, nosotros opinamos que aunque es un concepto reconocido por muchos investigadores, todavía sigue restringido a las fronteras latinoamericanas. En cambio, los otros términos tienden a adquirir cada vez más características globales. Principalmente en tiempos de la colonización, se llamaba «criollos» a los hijos de los españoles que nacieron en América. Posteriormente, según Sabine Hofmann (1997: 74) el término criollización se utilizó para calificar a las lenguas que surgían del contacto entre europeos y africanos en el contexto sociocultural de las plantaciones. Y después, dentro de su ámbito cultural fue considerado como un proceso continuo que se produce en un determinado espacio y tiempo. La transculturación de origen caribeño (el término fue acuñado por el sociólogo cubano Fernando Ortiz en la década de 1940) ${ }^{4}$ define el proceso que ocurre cuando dos o más culturas comparten y mezclan lo propio de sus culturas. Debemos apuntar que el proceso de transculturación se produce tanto voluntaria como involuntariamente. Pero al final resulta que nos encontramos con una cultura nueva, con influencias de las culturas que se interrelacionan. En este orden de cosas, debemos hablar todavía de dos nociones más: aculturación y asimilación.

Según el diccionario de la Real Academia Española (2001), aculturación significa «recepción y asimilación de elementos culturales de un grupo por parte de otro». En otras palabras sería adaptarse a una nueva cultura ${ }^{5}$. Este proceso resulta ser más profundo si se desarrolla en el país meta, en el país donde sus habitantes hablan la lengua que uno quiere aprender ${ }^{6}$. Entonces acontece un tipo de traspaso a una cultura

4 Véase Walter (1995, 170: 35).

5 Véase también las reflexiones acerca de la aculturación de Ezequiel Ander Egg (1995) que es autor del «Diccionario de Trabajo Social».

${ }^{6}$ En tiempos en que los medios de comunicación desarrollan un papel predominante podemos decir que en este caso se produce también un tipo de aculturación. Sin duda, los medios de comunicación presentan los perfiles culturales que una «sociedad perfecta» debería tener o en poco tiempo 
extranjera y en su fase final podemos hablar de la asimilación de ésta como la propia. El problema que ocurre es que adaptando otra cultura de la sociedad que es en cierto modo dominante, a veces de manera totalmente inconsciente, uno puede perder su propia identidad. Estas afirmaciones demuestran que en la enseñanza de lenguas se debe incluir cuestiones relacionadas con los riesgos y las consecuencias de los encuentros interculturales.

En la enseñanza de idiomas se habla de cuatro enfoques relacionados con el aprendizaje cultural (Risager, 1998: 242-254):

(1) el enfoque de la cultura extranjera, que va perdiendo terreno desde los años ochenta;

(2) el enfoque intercultural, que ha ocupado el lugar del enfoque de la cultura extranjera y que es el más vigente hoy en día;

(3) el enfoque multicultural, que aparece en los años ochenta pero que todavía ocupa un lugar marginal;

(4) el enfoque transcultural, que está empezando a surgir como consecuencia de la internacionalización.

El primer enfoque llamado el enfoque de la cultura extranjera, se basa en el concepto de la única cultura asociada a un pueblo e idioma específicos. Ocurre que principalmente no se estudia el país nativo del estudiante, evitándose simultáneamente el aprendizaje sobre las relaciones que puedan existir entre el país objeto de estudio y el del estudiante.

El segundo enfoque intercultural acepta las relaciones culturales entre el país objeto de estudio y el país del estudiante. Este concepto considera la interacción en el sentido de la comparación entre las dos culturas, también pone mucha atención en el intento por conocerse, entenderse y reconocerse. En el enfoque de la cultura extranjera solamente nos basamos en la cultura del país objeto, en cambio aquí también se estudia la cultura nativa del estudiante. También es bien vista su postura crítica respecto a la otra cultura.

El enfoque multicultural analiza el encuentro de diferentes culturas que existen en el país de estudio y presenta la diversidad étnica y lingüística de diferentes grupos sociales del país del estudio. En una sociedad multicultural la lengua oficial del país para algunos puede ser la primera lengua y para otros que viven dentro de la misma sociedad será la segunda, o incluso la lengua extranjera (por ejemplo, el caso de los vascos o los catalanes que hablan español como si fuera su primera lengua).

El enfoque transcultural se basa en la comunicación entre diferentes culturas. Se usa la lengua como «lingua franca» cuando ninguno de los hablantes tiene como

adquirir. La gente al recibir todo tipo de informaciones a través de estos medios a veces actúa y se expresa tal como presentan estas informaciones, sobre todo transmitidas por la televisión que difunde por todo el mundo las costumbres y paradigmas de la cultura de Occidente. En estas condiciones la aculturación parece ser inevitable y es difícil decir si es ya un proceso negativo o todavía sigue siendo de alguna manera positivo. 
primera lengua la lengua en cuestión. Por ejemplo los españoles, los polacos y los franceses que se comunican a través de la lengua inglesa en Estados Unidos. Así que, aquí también se trata de la complejidad cultural y lingüística, sin embargo en un encuentro transcultural participan por lo menos tres culturas distintas, entre ellas aparece la cultura del país objeto.

En definitiva, en el caso de la enseñanza de lenguas extranjeras centraremos nuestra atención sobre todo en el enfoque intercultural, donde se realiza un encuentro de dos culturas a base de respeto mutuo y correctas interpretaciones de lo diferente, en comparación con la propia cultura.

\section{EL CONCEPTO DE LA IDENTIDAD EN LA EDUCACIÓN INTERCULTURAL}

En referencia a las relaciones interculturales debemos tener en cuenta la importancia que desempeña la identidad propia de los interlocutores. El término de la identidad refleja sobre todo la visión de uno mismo que deriva del proceso de la socialización cultural, ética y de género tanto a nivel personal como social (Ting-Toomey, 1999: 2829). RAE (2001) define el término «identidad» como «conjunto de rasgos propios de un individuo o de una colectividad que los caracterizan frente a los demás». No cabe ninguna duda de que el modo de ver y percibir las cosas que nos rodean en un contexto social diferente está inseparablemente ligado a la identidad.

Michael Byram y Michael Fleming (2001:15) observan que cada persona tiene un número de identidades sociales, o sea, grupos sociales «a los que pertenecen, y culturas, prácticas, creencias y valores a los que se adhieren. Cuál de estas identidades va a ser dominante en cada interacción depende de los variados factores que intervienen en una situación: el idioma que se emplea, la relación con la otra persona, cómo se identifican los participantes». Los autores subrayan que cada contacto internacional se relaciona con distintas culturas e identidades nacionales. Y precisamente, por eso, en la enseñanza de lenguas se pone tanto interés en el análisis adecuado de los ya mencionados estereotipos. Lo que nosotros proponemos es una aproximación hacia otra cultura a través de la cual cada uno conserva su identidad cultural, sus costumbres, etc. Al mismo tiempo hay que valorar las relaciones que uno tiene con la sociedad de acogida. Esa sería la integración que correspondería a las tendencias actuales en la educación intercultural.

En opinión de Pilar García (1999), el concepto de la educación intercultural puede ayudar a superar el marco de la identidad cultural de un hombre, de tal manera que no renuncie a sus señas de identidad. La autora ${ }^{7}$ sostiene que:

\footnotetext{
${ }^{7}$ Cabe precisar que Pilar García (1999) en sus observaciones se refería sobre todo la situación de los inmigrantes en España. Sin embargo, creemos que conviene ampliar esta visión hacia todo tipo de contactos interculturales.
} 
El objetivo es conseguir que la diversidad no sea objeto de marginación manteniendo las distintas identidades culturales. Los esfuerzos dentro y fuera del aula han de encaminarse a procurar igualar las oportunidades sociales fuera de los grupos culturales. A través del conocimiento y valoración de las diferencias culturales se desarrolla el pluralismo cultural en la sociedad (García, 1999: 114).

La misma autora añade que la integración con la sociedad de acogida debería realizarse por medio de los siguientes pasos:

- conocer e identificarse con la cultura del país de acogida,

- llegar a un acercamiento entre ambas culturas, para posibilitar el análisis del comportamiento del otro y situarlo dentro de sus parámetros culturales,

- facilitar la adaptación de los inmigrantes para prevenir su posible marginación (García, 1999: 115).

Como conclusión debemos apuntar que en el proceso de la aculturación (en sentido negativo) uno puede hasta cambiar su identidad. Por eso la mayoría de los especialistas está de acuerdo en que la educación intercultural puede integrar socialmente al extranjero sin haber renunciado a su identidad nativa. En este caso el interculturalismo elige la integración porque es mutua y exige sobre todo la postura de respeto de ambos lados, unida a la sensibilización hacia las diferencias. Estamos de acuerdo con González Piñeiro, Guillén Díaz y Manuel Vez (2010: 229) en que «en el uso y aprendizaje de lenguas, las interacciones comunicativas implican una apertura a otras personas y, por tanto, a otras culturas que ponen en juego el componente cultural individual». Hay que añadir, siguiendo las palabras de los autores mencionados arriba, que la educación multicultural consistiría en el aprendizaje acerca de los diversos grupos culturales, ahondando en las diferencias culturales y, con el mismo énfasis, en el reconocimiento e identificación de las similitudes culturales. En nuestra opinión, estas dos actitudes, también ingredientes de la competencia intercultural ${ }^{8}$, son imprescindibles a la hora de realizar un encuentro intercultural eficaz.

\section{EL HABLANTE INTERCULTURAL Y UNA NUEVA VISIÓN PARA LA ENSEÑANZA DE LENGUAS: COMPETENCIA INTERCULTURAL Y PLURILINGÜE}

Cuando el alumno aprende una lengua extranjera se transforma en un intermediario o mediador (Byram, 1995) o actor intercultural (Oliveras, 2000) que además de conocimientos lingüísticos dispone de la llamada conciencia cultural. Según Byram y Fleming (2001), el hablante intercultural es alguien que tiene el conocimiento de una o, preferiblemente unas cuantas culturas e identidades sociales y también tiene la capacidad de descubrir y relacionarse con gente nueva de otros contextos para los cuales no ha sido preparado directamente. El hablante intercultural es tam-

\footnotetext{
${ }^{8}$ La noción de la competencia intercultural la explicamos en el punto 3 de este texto.
} 
bién alguien que aprende a ser independiente del profesor y también se da cuenta de los límites de lo que se puede conseguir en la clase (Holec, 1981). Pero lo que es más importante es que el hablante intercultural es una persona que tiene la habilidad de relacionarse con los «otros», acepta las perspectivas y percepciones del mundo de estos y es consciente de que puede evaluar y percibir el mundo de diferente manera (Byram, 1997).

Para transformarse en hablante intercultural el alumno debe contar con el apoyo del profesor y con los materiales que tiene a su disposición, especialmente los manuales. Por eso al principio el papel de mediador intercultural lo van a desarrollar el profesor y los materiales didácticos, que deberían ayudar al estudiante a interpretar las expresiones, intenciones y expectativas de los hablantes nativos. Belén Muñoz López (1998: 604) enumera los objetivos que se plantea a la hora de realizar una metodología determinada empleada en el aula con los chicos y chicas inmigrantes. Consideramos que estas observaciones se pueden tener en cuenta también durante la clase de lengua extranjera. En los primeros niveles de aprendizaje la autora señala los siguientes planteamientos:

- el desarrollo de la capacidad del alumno para comunicarse ${ }^{9}$,

- el lenguaje debe ser auténtico, cotidiano y adaptado al contexto,

- el componente sociocultural facilita la integración en el nuevo entorno social por medio de la comprensión de los distintos códigos de conducta y normas sociales.

En la época de la globalización cada vez más se apunta a que cada representante de una u otra cultura tiene derecho a ser diferente. La pregunta que nos planteamos es la siguiente: ¿qué puede hacer el profesor de lengua extranjera para que el alumno valore y respete las diferencias que puedan resultar muy chocantes para él? Estas reflexiones nos llevan a la noción de la competencia intercultural.

Aunque cada vez más científicos desarrollan nuevas definiciones de la competencia intercultural, todavía no existe una definición que pueda satisfacer a todos los especialistas en didáctica. En España, por ejemplo, la competencia intercultural aparece al mismo tiempo que proliferan los estudios sobre educación intercultural y también cuando la didáctica de las lenguas tiende a relacionarse con disciplinas tales como la antropología, la psicología o la pedagogía (Trujillo Sáez, 2001). Obviamente, el concepto de competencia intercultural está basado en la teoría de la competencia comunicativa, pero la consideramos como «un avance». Hasta los años 80, entre los elementos constituyentes de la competencia comunicativa aparecían los elementos relacionados con los aspectos sociales, socioculturales o sociolingüísticos (Hymes, 1972; Canale, Swain, 1980). En los últimos años ha aparecido un nuevo concepto de competencia comunicativa que acoge todas estas subcompetencias y que adquiere el nombre de competencia cultural o competencia intercultural.

${ }^{9}$ Muñoz López (1998: 604) considera que en el proceso de comunicación cometer errores es algo inevitable, a partir del cual se debe progresar. 
El término de competencia intercultural, según Ángels Oliveras (2000), va más allá del concepto de la competencia sociocultural como parte integrante de la competencia comunicativa porque el acento en esta competencia cae en el aspecto cultural en la enseñanza de una lengua extranjera. «Se trata de una cuestión de conocimiento, actitudes y destrezas» (Oliveras, 2000: 32). Hablando del desarrollo del concepto de la competencia intercultural, no podemos omitir el anterior nacimiento de la competencia cultural, apuntando que se trata del estudio solamente de una cultura, sin hacer referencias a la propia cultura ${ }^{10}$.

Byram (1995), uno de los investigadores más relevantes que estudia la relación entre el aprendizaje de lenguas y el estudio de otras culturas, al elaborar su definición de competencia intercultural, ha recurrido a las investigaciones sobre las competencias (sociolingüística, estratégica, sociocultural) y ha definido la competencia intercultural como componente de:

- savoir-être (cambio de actitud, actitudes),

- savoirs (adquisición de nuevos conceptos, conocimientos),

- savoir-faire (aprendizaje a través de la experiencia, destrezas).

Más tarde, el autor desarrolla el concepto añadiendo las destrezas relacionadas con la interpretación y la educación. Esta última se basa en disponer de la habilidad de evaluar de una manera crítica y a base de unos criterios explícitos, las perspectivas, prácticas y productos tanto de la suya propia como de las otras culturas y países (Byram, 1997: 63).

Byram, Nichols y Stevens (2001) añaden que cada mediador/hablante intercultural debería disponer de actitudes interculturales (en francés: savoir-être) que se basan sobre todo en tener la curiosidad y habilidades de apreciar otras culturas. Además de las actitudes se recurre a los conocimientos (en inglés: knowledge ${ }^{11}$. En principio no se trata de los conocimientos acerca de una cultura específica, sino de cómo los grupos e identidades sociales funcionan. Hay que subrayar que tanto los profesores de lenguas extranjeras como sus estudiantes nunca dispondrán de la totalidad de los conocimientos culturales posibles. La enorme diversidad cultural tanto a nivel nacional, regional o incluso familiar imposibilita el acceso a todas las informaciones que caracterizan una determinada cultura. Por eso es necesario que los profesores se concentren más en las habilidades (o sea skills of discovery and interaction) que a largo plazo ayudarán a sus estudiantes a buscar las informaciones que necesiten y luego integrarse con los miembros de otra cultura. Por último, los mediadores deben ser conscientes de sus propios valores y de cómo estos valores pueden influir en los valores de otra gente (critical cultural awareness). El papel del profesor debería comprender el desarrollo

10 Véase las anteriores reflexiones acerca del enfoque cultural.

11 Lo que Byram (1997) entiende bajo el concepto de Knowledge, Oliveras (2000) lo nombra conocimientos y el Marco Común de Referencia para las Lenguas de Consejo de Europa (2002) utiliza la noción del conocimiento declarativo, o sea el saber; de la misma manera: Skills serán destrezas y Attitudes: actitudes. 
de las habilidades, actitudes y la conciencia de los valores de la misma manera que la presentación de los contenidos culturales. Asimismo, la competencia intercultural puede ser definida como la «disposición de las habilidades para conocer todo lo que caracteriza a la otra cultura y después saber ejecutar las actitudes apropiadas y eficaces durante un encuentro intercultural. Precisamente esta interpretación debería, en nuestra opinión, ser enseñada y promovida entre los profesores, alumnos y autores de los materiales de lenguas extranjeras» (Spychała, 2009: 145).

Como ya hemos apuntado, las relaciones interculturales se caracterizan por mucha diversidad tanto cultural como lingüística, «lo cual es absolutamente normal si tomamos en cuenta que la realidad cultural de los individuos encierra toda una gama de variantes dialectales, de formas de vida, de costumbres, tradiciones y creencias, es decir, de cosmovisiones» (Solís, 2012: 182). Estas consideraciones nos llevan al nuevo concepto de la competencia plurilingüe y pluricultural. Según el Marco Común de Referencia para las Lenguas (MCER) del Consejo de Europa (2002: 167) la competencia plurilingüe y pluricultural hace referencia a la capacidad de utilizar las lenguas para fines comunicativos y de participar en una relación intercultural en la que una persona, en cuanto agente social, domina - con distinto grado-varias lenguas y posee experiencia de varias culturas. Debemos añadir que el plurilingüismo no es lo mismo que el multilingüismo, ya que éste último «se limita a describir la coexistencia de varias lenguas o variedades intralingüísticas en una zona determinada, el del plurilingüismo ha de entenderse como la capacidad de los individuos de interrelacionar los conocimientos que van adquiriendo de cada una de las distintas lenguas» (González Piñeiro, Guillén Díaz y Manuel Vez, 2010: 18).

\section{EL MARCO COMÚN DE REFERENCIA PARA LAS LENGUAS (MCER) Y LA COMPETENCIA COMUNICATIVA INTERCULTURAL EN LA ENSEÑANZA DE LAS LENGUAS EXTRANJERAS}

El Marco Común de Referencia para las Lenguas (MCER) es uno de los proyectos clave que forman parte de la política lingüística del Consejo de Europa (2002). En la introducción de este documento leemos que el MCER proporciona una base común para la elaboración de programas de lenguas, orientaciones curriculares, exámenes, manuales, etc. en toda Europa. Describe de forma integradora lo que tienen que aprender los estudiantes de las lenguas con el fin de utilizar una lengua para comunicarse, así como los conocimientos y destrezas que tienen que desarrollar para poder actuar de manera eficaz. También se toma en cuenta el contexto cultural de la lengua. El MCER analiza sobre todo diferentes niveles de dominio de la lengua con el fin de verificar el progreso de los alumnos. Uno de los mayores objetivos de este proyecto es llegar a un sistema unificado de educación de las lenguas en toda Europa. Entre otras aportaciones el documento establece los niveles $(\mathrm{A}, \mathrm{B}, \mathrm{C})$ de dominio de una lengua extranjera. 
Al ver el contenido del MCER podemos decir que se dedica bastante espacio a los problemas relacionados con la cultura en la relación estricta con la lengua. «La lengua no es sólo un aspecto importante de la cultura, sino un medio de acceso a las manifestaciones culturales» (MCER, 2002: 6).

Además del sistema de niveles, hay que subrayar que el MCER (2002: 99-106) especifica tres competencias comunicativas relacionadas con la didáctica de la lengua extranjera:

- competencia lingüística,

- competencia sociolingüística,

- competencia pragmática (que incluye a su vez las competencias discursivas, funcionales y organizativas).

Debido al tema de nuestro estudio nos detendremos con más detalle en la competencia sociolingüística y la competencia sociocultural. La competencia sociolingüística comprende el conocimiento y las destrezas necesarias para abordar la dimensión social del uso de la lengua, especialmente los marcadores lingüísticos de relaciones sociales, las normas de cortesía, las expresiones de la sabiduría popular, las diferencias de registro, el dialecto y el acento. Cada uno de estos tres componentes consta de cuatro elementos llamados competencias generales menos relacionadas con la lengua:

(1) Conocimiento declarativo (saber) / Declarative knowledge (savoir),

(2) Las destrezas y las habilidades (saber hacer) / Skills and know-how (savoir-faire),

(3) La competencia existencial (saber ser) /'Existential' competence (savoir-être),

(4) La capacidad de aprender (saber aprender) / Ability to learn (savoir-apprendre).

En relación al (1) conocimiento declarativo los autores del documento proponen tres elementos: el conocimiento del mundo, el conocimiento sociocultural y la conciencia intercultural. Entre los elementos que describen una sociedad se citan:

- la vida diaria,

- las relaciones personales,

- los valores, las creencias y las actitudes,

- el lenguaje corporal: el conocimiento de las convenciones que rigen el comportamiento de los miembros de un país,

- las convenciones sociales (por ejemplo, respecto a ofrecer y recibir hospitalidad),

- el comportamiento ritual.

Los autores del MCER apuntan que la conciencia intercultural es la que incluye la conciencia de la diversidad regional y social en ambos mundos «que se enriquece con la conciencia de una serie de culturas más amplia de la que conlleva la lengua materna y la segunda lengua, lo cual contribuye a ubicar ambas en su contexto» (MCER, 2002: 101). 
(2) Las destrezas y habilidades (savoir-faire) interculturales son las capacidades de relacionar entre sí la cultura de origen y la cultura extranjera. Entre otras capacidades, los autores del MCER incluyen:

- la sensibilidad cultural y la capacidad de identificar y utilizar una variedad de estrategias para establecer contacto con personas de otras culturas,

- la capacidad de cumplir el papel de intermediario cultural y de abordar con eficacia los malentendidos interculturales y las situaciones conflictivas,

- la capacidad de superar relaciones estereotipadas.

Los alumnos, además de sus conocimientos y sus habilidades, deben tener en cuenta las características personales (las actitudes, las motivaciones, los valores, las creencias, los estilos cognitivos) que sin duda afectan a la comunicación intercultural ${ }^{12}$, lo que se denomina como (3) la competencia existencial (savoir-être), o sea, saber ser. Y (4) la capacidad de aprender (savoir-apprendre) permite al estudiante observar y participar en nuevas experiencias y después incorporar nuevos conocimientos a los existentes, haciendo al mismo tiempo la modificación cuando sea necesario.

Como vemos, el MCER (2002) establece una base de recursos que deben ser aprovechados en el aprendizaje de la lengua extranjera y que son muy similares a las ideas de otros autores antes descritas. Se subraya que uno de los objetivos fundamentales de la educación en la lengua es el impulso del desarrollo favorable de la personalidad del alumno y de su sentimiento de identidad, como respuesta a la enriquecedora experiencia que supone enfrentarse a lo diferente en los ámbitos de la lengua y de la cultura. Esta sería la tarea de los profesores y de los alumnos: reintegrar estas cuestiones de manera fructífera (MCER, 2002: 1)

Así como lo refleja este documento europeo, cualquier forma de uso y de aprendizaje-enseñanza de la lengua abarca las acciones de las personas (los individuos sociales) que desarrollan una gama de competencias, que son las competencias comunicativas unidas con las denominadas competencias generales. Los estudiantes deben usar estas competencias (destrezas y habilidades) en diferentes contextos con el fin de realizar las tareas y las actividades durante distintas situaciones comunicativas.

Lo que sorprende es que los autores del MCER (2002) prefieran hablar de las competencias generales en vez de la competencia intercultural. Pero sin duda, estas competencias generales cubren todos los elementos que constituyen la competencia intercultural. Una de las cosas que llama la atención es la importancia que se da a las habilidades y destrezas respecto a la competencia intercultural más que al domino de los conocimientos sobre la cultura extranjera. Hasta ahora eran y siguen siendo los conocimientos que se evalúan en los programas de la enseñanza de lenguas extranjeras. El MCER recuerda que en la práctica serán los profesores, los autores de los

${ }^{12}$ Aconsejamos leer el libro de Edward T. Hall (1984) «Poza kulturą», donde el autor demuestra que para entender la otra cultura debemos dejar de lado nuestras experiencias culturales, no debemos dar opiniones solo desde nuestra perspectiva cultural. 
materiales de la enseñanza de la lengua extranjera los que, ofreciendo una gama de conocimientos (savoir) al alumno, deben al mismo tiempo desarrollar las competencias necesarias ( habilidades y actitudes) que ayudarán al alumno a actuar como intermediario cultural.

Sin duda, al proporcionar una base de directrices se tuvo cuenta los límites con los cuales se encuentran los profesores y los autores de los materiales a la hora de proporcionar todo tipo de conocimientos sobre la cultura extranjera durante la clase. Como ya lo hemos dicho, es imposible enseñar / presentar durante la clase todos los contenidos culturales de un país meta. Basta con ver la diversidad cultural que está detrás de la lengua española. Por eso se requiere que el alumno desarrolle unas habilidades y capacidades que le permitan funcionar en una cultura nueva sin disponer previamente de todos elementos que forman parte de su «conocimiento declarativo». De esta manera, gracias a las capacidades interculturales el alumno puede aprender y conocer la otra cultura en cualquier momento fuera del aula.

\section{CONCLUSIONES}

En los tiempos de la globalización, crecen cada vez más los contactos internacionales. Recordemos que las culturas pueden ser diferentes no sólo entre los países sino también dentro del país, la misma empresa o incluso familia. Cada ser humano tiene su propia cultura que puede ser: histórica, geográfica, artística, religiosa, ética, moral, etc. Esas situaciones en las que se encuentran los participantes - hablantes interculturales, mediadores culturales - requieren de ellos una formación intercultural. Lo que quiere decir que cada uno de ellos debería estar ya educado y preparado.

Con los contenidos presentados en este trabajo hemos pretendido mostrar que en el mundo actual si no tenemos en cuenta las herramientas de la comunicación intercultural podemos perjudicar cada acto comunicativo. Consideramos que es necesario establecer de manera más rigurosa y unificada las pautas didácticas para la enseñanza de lenguas extranjeras desde la perspectiva intercultural. Por medio de la llamada competencia intercultural se puede llegar a un encuentro de igualdad entre distintas culturas sin olvidarse de la propia identidad. Por eso, en nuestra opinión, hay que hablar de la conciencia y la comprensión de los comportamientos de los otros desde las primeras clases de lengua extranjera.

BIBLIOGRAFÍA

Ander-EgG, Ezequiel (1995): Diccionario de trabajo social. Buenos Aires: Lumen Argentina.

Bravo Bosch, María Cristina (1998): «Catálogo de necedades que los europeos se aplican mutuamente, o cómo llegar al siglo XXI sin repetir los tópicos de los otros XX». In: Ángela CeLIS, José Ramón Heredia [eds.], Lengua y cultura en la enseñanza de español a extranjeros, Actas 
del VII Congreso Internacional de ASELE. Cuenca: Ediciones de la Universidad de Castilla-La Mancha,143-149.

Byram, Michael; Nichols Adam \& Stevens David (2001): «Introduction». In: Michael Brram; Adam Nichols \& David Stevens [eds.], Developing Intercultural Competence in Practice. Clevedon: Multilingual Matters LTD, 1-8.

Byram, Michael; Fleming Michael (1998, 2001): «Culture and language learning». In: Michael Byram, Michael Fleming [eds.], Language Learning in Intercultural Perspective. Cambridge, CUP, 1-15.

BYrAm, Michael (1995): «Acquiring Intercultural Competence. A Review of Learning Theories». In: Lies Sercu [ed.], Intercultural competence, Vol. I, 53-70.

Byram, Michael (1997): Teaching and Assessing Intercultural Communicative Competence. Clevedon: Multilingual Matters LTD.

Canale, Michael; Swain Michael (1980): «Theoretical bases of communicative approaches to second language teaching and testing». Applied Linguistics, 1: 1-47.

Consejo De Europa (2002): Marco Común Europeo de Referencia para las Lenguas: Aprendizaje, Enseñanza, Evaluación. Madrid. Anaya. Disponible en http://cvc.cervantes.es/ensenanza/biblioteca_ele/marco/cvc_mer.pdf (español) [29.06.2013].

GARCíA, Pilar (1999): «El enfoque intercultural en la enseñanza de español a inmigrantes». In: Lengua y Cultura en el aula de E/LE, CARABELA 45. Madrid: SGEL, 107-119.

GonzÁlez Piñeiro, Manuel; Guillén Díaz, Carmen \& Vez Manuel José (2010): Didáctica de las lenguas modernas. Competencia plurilingüe e intercultural. Madrid: Editorial Síntesis.

GudYKunst, William; Kıм, Young Yun (1984): Communicating with strangers: an approach to intercultural communication. New York: Random House.

HaLl, Edward Twichell (1959): The silent language. New York: Doubleday.

Hall, Edward Twichell (1984): Poza kultura. Warszawa: PWN.

Hofmann, Sabine (1997): «Transculturación and Creolization: Concepts of Caribbean Cultural Theory». In: Richard A. Young [ed.], Latin American Postmodernism. Amsterdam: Rodopi, 68-79.

Holec, Henri (1981): Autonomy and foreign Language Learning. Oxford: Pergamon.

Hymes, Dell Hathaway (1972): «On Communicative Competence. In: J.B. Pride, Janet Holmes, Sociolinguistics. Selected Readings. Harmondsworth: Penguin, 269-293.

IgLESIAS CASAL, Isabel (1998): «Diversidad cultural en el aula de E/LE: la interculturalidad como desafío y como provocación» In: Francisco Moreno, María GiL \& Kira Alonso [eds.], La enseñanza del español como lengua extranjera: del pasado al futuro. Actas del VIII Congreso Internacional de ASELE. Madrid: Universidad de Alcalá de Henares, 463-472.

Kramsch, Claire (1998): «The privilege of the intercultural speaker». In: Michael Byram, Michael Fleming [eds.], Language Learning in Intercultural Perspective. Approaches through drama and etnography. Cambridge: CUP, 23-38.

Matsumoto, David; JuAng, Linda (2007): Psychologia międzykulturowa. Gdańsk: GWP.

MuÑoz LóPEz, Belén (1998): «La enseñanza del español como segunda lengua para el alumnado inmigrante en los niveles obligatorios de enseñanza (E.P. Y E.S.O.)». In: Francisco MoReno, María Gil \& Kira Alonso [eds.], La enseñanza del español como lengua extranjera: del pasado al futuro. Actas del VIII Congreso Internacional de ASELE. Madrid: Universidad de Alcalá de Henares, 599-607.

Murdoch, Anna (1999): Wspólpraca z cudzoziemcami w firmie. Warszawa: Poltext.

OlIVERAS, Ángels (2000): Hacia la competencia intercultural en el aprendizaje de una lengua extranjera. Madrid: Edinumen.

Real Academia Espańola (2001): Diccionario de la lengua española. 22 a edición. Madrid: Espasa - Calpe. 
Risager, Karen (1998): «Language teaching and the process of European integration». In: Language Learning in Intercultural Perspective. Approaches through drama and etnography. Cambridge: CUP, 242-254.

Rodrigo Alsina, Miguel (1999): La comunicación intercultural. Barcelona: Anthropos.

Solís, C. Litza (2012): «La enseñanza de la competencia intercultural en el aula de E/LE: Consideraciones didácticas para programas de inmersión lingüíistico-cultural (PILC)». Revista Nebrija de Lingüíistica Aplicada, 11 (6): 174-192.

SpychaŁA, Małgorzata (2009): «La importancia de la competencia intercultural en la interpretación de las diferencias culturales en la enseñanza del español como lengua extranjera». In: Agnieszka KŁosińsKa-Nachin, Marek BARAn [eds.], Entrecruces. Estudios Hispánicos e Hispanoamericanos. Łodź: Wyższa Szkoła Studiów Międzynarodowych w Łodzi, 143-157.

Szurmuk, Mónica; Mckee Irvin, Robert (2009): Diccionario de Estudios Culturales Latinoamericanos. México: Instituto Mora. Siglo XXI editores, 119.

Ting-Toomey, Stella (1999): Communicating Across Cultures. New York: The Guilford Press.

TrujILlo SaÉz, Fernando (2001): «Objetivos en la enseñanza de lenguas extranjeras: De la competencia lingüística a la competencia intercultural», Comunicación presentada en el Congreso Nacional Inmigración, Convivencia e interculturalidad, organizado en Ceuta por el Instituto de Estudios Ceutíes.

Van Den Berg-Eldering, Lotty (1987): «Conférence finale. Rapport général». DECS/EGT, 86. Rotterdam - Estrasburgo: Consejo de Europa.

WaLter, Mignolo (1995): «Occidentalización, Imperialismo, Globalización: herencias coloniales y teorías poscoloniales». Revista Iberoamericana, 170-171: 27-40. 